\title{
ANÁLISE DA CONFIABILIDADE DE UMA INDÚSTRIA DE ALIMENTOS
}

Israel de Carvalho Lino; Carla Patrícia Santos Oliveira Rodrigues Esquerre²; Ângelo Márcio Oliveira Sant'Anna²

${ }^{1}$ Escola Politécnica da Bahia-UFBA; R. Aristídes Novis- Federação; Salvador /Ba; israel.lino@outlook.com

${ }^{2}$ Escola Politécnica da Bahia-UFBA; R. Aristídes Novis- Federação; Salvador /Ba;

Resumo: $O$ presente trabalho tem por objetivo analisar as principais medidas da confiabilidade e o tempo médio até a falha do maquinário que mais implica nas falhas no processo de produção de salgadinhos derivado de milho. Foi utilizado teste de hipótese Anderson-Darling para avaliar a melhor distribuição dos dados coletados, evidenciando a distribuição lognormal como a que melhor modelava aos dados e para tal foi utilizado o software Minitab v.17. Os resultados apresentaram um indicador de MTTF em 2,5 dias e $R(t)=0,47$. Observa-se que o equipamento empacotadora é dependente das ações da manutenção possuindo apenas $47 \%$ de chances de não apresentar uma falha quando em funcionamento.

Palavras-Chave: Confiabilidade; lognormal; MTTF; teste de hipótese.

\section{CONFIABILITY ANALISYS A FOOD FACTORY}

\begin{abstract}
The present work has as objective to analyze the main reliability measures for packing equipment, used in the process for production of chips derived from corn. The Anderson-Darling hypothesis test was used to evaluate the best distribution of the collected data, showing that the lognormal distribution was the best model for the data and was used Minitab v.17 software. The results showed an MTTF indicator at 2.5 days and $\mathrm{R}(\mathrm{t})=0.47$. Furthermore, the packaging equipment is dependent on maintenance actions having only $47 \%$ chance for not having a failure when in operation.
\end{abstract}

Keywords: Reliability; lognormal; MTTF; hypothesis test. 


\section{INTRODUÇÃO}

A economia globalizada e a busca incessante da recuperação da crise econômica, tem exigido das empresas maior grau de gestão, tendo as organizações buscado novas ferramentas de gerenciamento que aponte para uma maior competitividade através da qualidade e produtividade dos produtos, processos e serviços [1].

O alvo de uma melhor gestão é sempre buscar maior lucratividade, redução de custos e assim promover o crescimento da empresa e a sua competitividade, em resumo, podemos simplificar que o objetivo é o aumento da produtividade. Neste sentido um crescimento da produtividade implica um melhor aproveitamento de funcionários, máquinas, da energia e dos combustíveis consumidos, da matéria-prima e assim por diante [2].

Dos fatores apresentados acima que implicam na vulnerabilidade do indicador de produtividade, será discutido a diante o impacto causado pelas falhas de maquinário, avaliando o cenário da gestão da manutenção centrada em confiabilidade (MCC). Os dados serão organizados em gráficos de Pareto para determinar o maior impacto por maquinário e por maior demanda de abertura de chamados, depois aplicar os dados nas funções de probabilidade para falhas passando a conhecer qual o modelo que apresenta o melhor comportamento dos dados e a partir daí aplicar os dados a função confiabilidade $\mathrm{R}(\mathrm{t})$ e MTTF.

Este artigo tem por objetivo analisar a confiabilidade e o tempo médio até a falha do maquinário que mais implica nas falhas da linha de produção estudada. Conhecendo estes números o departamento da manutenção poderá direcionar os seus esforços e ações para a correção nos pontos indicados no gráfico de Pareto para futuras medições dos indicadores e acompanhar a sua evolução.

\section{REFERENCIAL TEÓRICO}

\subsection{Manutenção Centrada em Confiabilidade}

O termo manutenção centrada em confiabilidade refere-se a um programa de manutenção planejada para retornar a capacidade produtiva inerente do equipamento [3]. O objetivo principal da manutenção é manter e melhorar a confiabilidade e a regularidade de operação do sistema produtivo [4].

A MCC incorpora novas técnicas de manutenção e monitoramento além de absorver métodos modernos de otimização estatística desenvolvidos pela engenharia de produção, sendo uma de suas vantagens o estabelecimento de uma forma estruturada para selecionar as atividades de manutenção [5].

A medida que o sistema produtivo apresenta menos falhas, aumenta a confiabilidade e a disponibilidade das máquinas e equipamentos. Para [6] um sistema em operação normal, as falhas são eventos aleatórios causados por aumento súbito de estres ou erro humano. 


\subsection{Principais indicadores e funções da Confiabilidade}

O tempo até a falha, medido a partir do momento que a unidade é colocada em funcionamento até a falha, é definida pela função:

$$
F(t)=P(T \leq t)=\int_{0}^{t} f(u) d u, t>0
$$

A confiabilidade, definida pela função:

$$
R(t)=\frac{n_{s}(t)}{n_{s}(t)+n_{f}(t)}=\frac{n_{s}(t)}{n_{0}}
$$

O risco, também conhecida como taxa de falha ou taxa de risco e está associado as condições em que a unidade está submetida. É definida pela função:

$$
h(t)=\lim _{\Delta t \rightarrow \infty} \frac{R(t)-R(t+\Delta t)}{R(t) \Delta t}=\frac{-R(t)}{R(t)}=\frac{f(t)}{R(t)}, t \geq 0
$$

O tempo médio até a falha ou MTTF do inglês (mean time of failure)

$$
M T T F=E(t)=\int_{0}^{+\infty} t f(t) d t
$$

\section{METODOLOGIA}

Os principais modelos utilizados para descrever funções de confiabilidade são as distribuições de probabilidade exponencial, Weibull, gamma, lognormal e normal $[4,2]$. Nas definições de análise de confiabilidade faz-se necessário determinar qual distribuição de probabilidade melhor se ajusta aos dados.

O presente trabalho se configura como um estudo de caso, quando envolve o estudo profundo e exaustivo de um ou poucos objetos de maneira que se permitam conhecimentos amplos e detalhados [8]. Foram coletados dados reais de uma empresa e aplicada a técnica da manutenção centrada em confiabilidade. Para os gráficos das funções de probabilidade de alguns modelos estatísticos foi utilizado o software Minitab v.17.

\subsection{A Empresa}

Localizada no Estado da Bahia com sede industrial em Salvador a empresa é líder na região nordeste na produção de derivados de milho, snacks ou mais popularmente conhecido como "salgadinhos". A unidade fabril é composta de 5 linhas de produção e possuem aproximadamente 200 funcionários atuando diretamente na produção. $A$ linha de produção estudada foi de "salgadinho" segue algumas definições que caracterizam a operação para funcionamento da linha.

a) Jornada de trabalho: segunda a sábado das $22 \mathrm{~h}$ às $06 \mathrm{~h}$ iniciando domingo $22 \mathrm{~h}$ e parando sábado $22 \mathrm{~h}$;

b) Três turnos de operação, noite, manhã e tarde;

c) A linha é composta por 4 maquinários; 
d) Atuam diretamente na linha 60 colaboradores sendo 20 por turno;

e) Jornada média de 25 dias de produção por mês;

f) O start da linha é feito aos domingos no turno da noite que inicia as $22 \mathrm{~h}$ sendo que é programado o tempo de $1 \mathrm{~h}$ para preparo das máquinas;

g) Todas as terças-feiras são celebradas um culto as $11 \mathrm{~h}$ para os funcionários e todos os colaboradores do turno da manhã, são liberados $1 \mathrm{~h}$ mais cedo para que possam participar. Aos sábados o turno da manhã encerra a operação da linha as $13 \mathrm{~h}$;

h) Aos sábados é feita a limpeza da linha, assim não há operação de produção no turno da tarde;

A Tabela 1 apresenta a jornada em horas diárias de cada turno de trabalho e define as horas de operação em produção da linha. É possível observar que a linha de salgadinho opera por 19,33h/dia.

Tabela 1 - Jornada de trabalho dos turnos

\begin{tabular}{|c|c|c|c|c|c|c|c|c|}
\hline \multicolumn{8}{|c|}{ HORAS DE TRABALHADAS POR DIA POR TURNO } & \multirow{2}{*}{$\begin{array}{c}\text { MÉDIA DE HORAS } \\
\text { TRABALHADAS POR } \\
\text { TURNO POR DIA }\end{array}$} \\
\hline TRUNO & SEG & TER & QUA & QUI & SEX & SAB & DOM & \\
\hline NOITE & 6 & 7 & 7 & 7 & 7 & 7 & 0 & 6,83 \\
\hline MANHÃ & 7 & 6 & 7 & 7 & 7 & 6 & 0 & 6,67 \\
\hline TARDE & 7 & 7 & 7 & 7 & 7 & 0 & 0 & 5,83 \\
\hline FÁBRICA & 20 & 20 & 21 & 21 & 21 & 13 & 0 & 19,33 \\
\hline
\end{tabular}

Na Tabela 2 são apresentados dados do tempo médio entre falhas, em horas por mês, dos maquinários que compõe a linha de produção de salgadinhos.

Tabela 2 - MTTF $(\mathrm{h})$ dos maquinários da linha de salgadinho

\begin{tabular}{|lc|c|c|c|c|c|c|}
\cline { 2 - 9 } \multicolumn{1}{c|}{} & JAN & FEV & MAR & ABR & MAI & JUN & JUL \\
\hline ESTEIRA DE ABASTECIMENTO & 136 & 11 & 82 & 9 & 11 & 6 & 9 \\
\hline EXTRUSORA & 367 & 217 & 22 & 29 & 32 & 35 & 96 \\
\hline EMPACOTADORA & 211 & 269 & 420 & 101 & 158 & 124 & 180 \\
\hline FORNO & 80 & 50 & 30 & 20 & 15 & 15 & 10 \\
\hline
\end{tabular}

Fonte: Autores

A Tabela 3 traz informação dos dados acumulados das quantidades de ordens de serviços de manutenção abertas a cada mês. A Tabela 4 traz uma informação importantíssima para o direcionamento das ações do departamento da manutenção, pensado no planejamento da carga de trabalho para cada hora homem disponível.

Tabela 3 - Número de ocorrências

\begin{tabular}{|l|r|r|r|r|r|r|r|}
\hline ESTEIRA DE ABASTECIMENTO & 31 & 46 & 28 & 19 & 13 & 15 & 18 \\
\hline EXTRUSORA & 39 & 24 & 10 & 25 & 20 & 9 & 20 \\
\hline
\end{tabular}


Fonte: Autores

A Tabela 4, a Figura 2 e a Figura 3 ilustram a priorização das ações do departamento de manutenção para os equipamentos que mais impacta em falhas e planejar o seu efetivo, hora homem quanto ao tipo de manutenção.

Observa-se que se justifica concentrar energia em atuar no equipamento empacotadora com forte atuação da equipe de manutenção mecânica, devido ao número de falhas no equipamento.

Tabela 4 - Número de chamados por tipo de manutenção

$\begin{array}{lccccccc} & \text { JAN } & \text { FEV } & \text { MAR } & \text { ABR } & \text { MAI } & \text { JUN } & \text { JUL } \\ \text { ELÉTRICA } & 15 & 22 & 15 & 19 & \mathbf{2 1} & \mathbf{3} & \mathbf{2} \\ \text { MECÂNICA } & 90 & 88 & 60 & 66 & 58 & 20 & 25 \\ \text { PENUMÁTICA } & 10 & 15 & 5 & 8 & 8 & 2 & 3 \\ \text { TOTAL } & 115 & 125 & 80 & 93 & 87 & 25 & 30\end{array}$

Figura 2 - Pareto do MTTF dos maquinários da linha de salgadinho

\begin{tabular}{|c|c|c|c|c|c|c|}
\hline \multirow{4}{*}{ 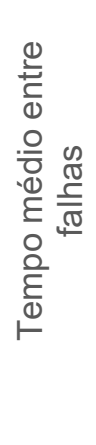 } & 2000 & 1677 & $85,76 \%$ & $94,68 \%$ & $102,11 \%$ & \multirow{4}{*}{$\begin{array}{l}120,00 \% \\
100,00 \% \\
80,00 \% \\
60,00 \% \\
40,00 \% \\
20,00 \% \\
0,00 \%\end{array}$} \\
\hline & & & 798 & & & \\
\hline & 500 & & & 264 & 220 & \\
\hline & & EMPACOTADORA & $\begin{array}{r}\text { EXTRUSORA } \\
\text { Maqu }\end{array}$ & $\begin{array}{l}\text { ESTEIRA DE } \\
\text { ABASTECIMENTO } \\
\text { inário }\end{array}$ & FORNO & \\
\hline
\end{tabular}

Fonte: Autores

Figura 3 - Pareto de priorização por tipo de manutenção

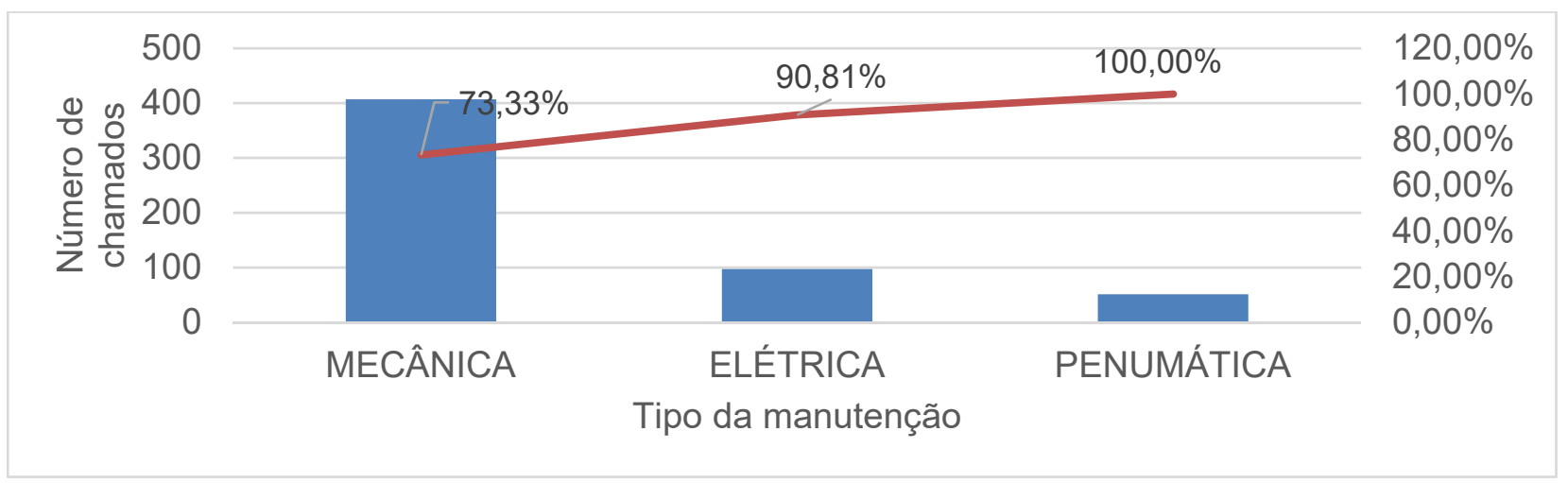

Fonte: Autores 
A Tabela 5 apresenta o MTTF(h) semanal da empacotadora da linha de salgadinhos, devido a análise da representação gráfica da figura 1 demonstrando a importância na priorização das ações na empacotadora.

\begin{tabular}{|c|c|c|c|c|}
\hline SEM 1 & SEM 2 & SEM 3 & SEM 4 & SEM 5 \\
\hline 40 & 35 & 45 & 51 & 40 \\
\hline SEM 6 & SEM 7 & SEM 8 & SEM 9 & SEM 10 \\
\hline 68 & 67 & 67 & 68 & 70 \\
\hline SEM 11 & SEM 12 & SEM 13 & SEM 14 & SEM 15 \\
\hline 130 & 100 & 120 & 50 & 20 \\
\hline SEM 16 & SEM 17 & SEM 18 & SEM 19 & SEM 20 \\
\hline 20 & 11 & 35 & 46 & 48 \\
\hline SEM 21 & SEM 22 & SEM 23 & SEM 24 & SEM 25 \\
\hline 30 & 35 & 37 & 40 & 13 \\
\hline SEM 26 & SEM 27 & SEM 28 & SEM 29 & SEM 30 \\
\hline 39 & 25 & 28 & 31 & 30 \\
\hline $\begin{array}{c}\text { SEM } 31 \\
\mathbf{2 7}\end{array}$ & & & & \\
\hline
\end{tabular}

Fonte: Autores

Para análise e definição do modelo utilizamos o teste de Anderson-Darling que serve para verificar o quão bem os dados seguem uma distribuição. Por meio da análise dos valores expressos na Tabela 6 . No teste de Anderson-Darling verificamos que a Lognormal é o modelo de probabilidade que melhor apresenta a distribuição dos dados.

Figura 4 - Gráfico de probabilidade para a falha

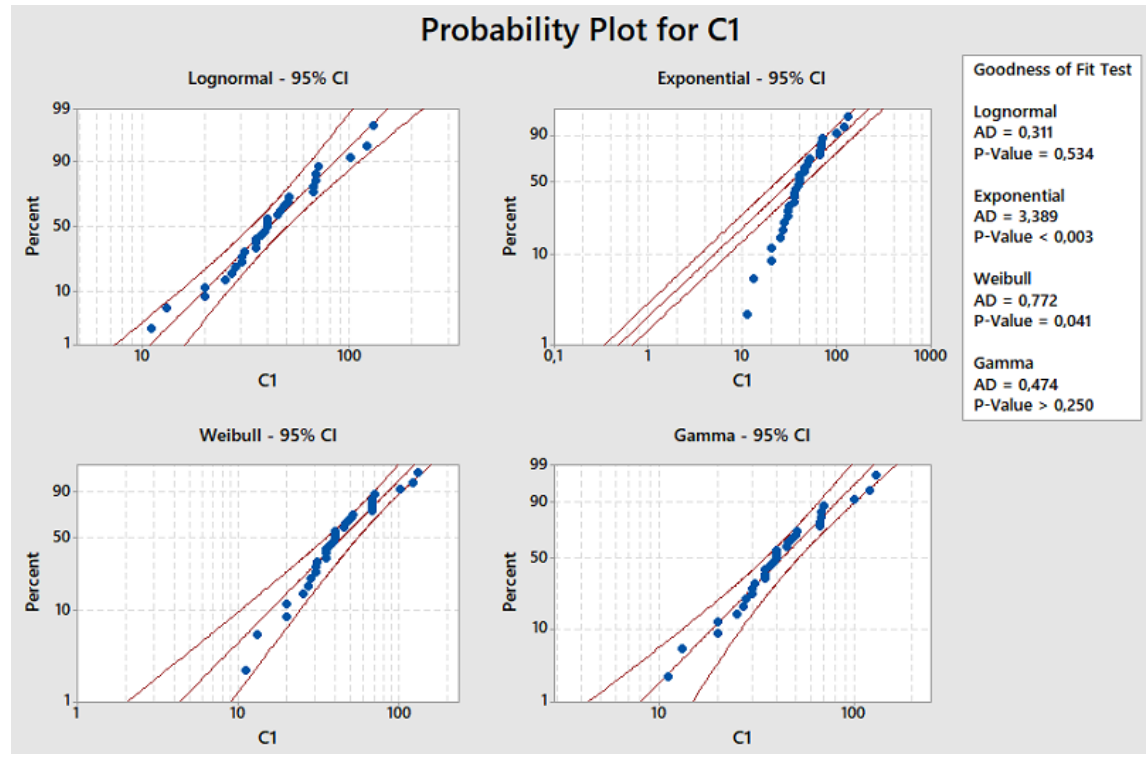

Fonte: Autores 


\section{\begin{tabular}{l|l} 
CIRCULAR ECONOMY & ECONOMIA CIRCULAR
\end{tabular}}

Tabela 6 - Valores da estatística de Anderson-Darling para várias distribuições

\begin{tabular}{|l|c|}
\hline \multicolumn{1}{|c|}{ Distribution } & AD \\
\hline Normal & 1,512 \\
\hline Box-Cox Transformation & 0,311 \\
\hline Lognormal & 0,311 \\
\hline 3-Parameter Lognormal & 0,305 \\
\hline Exponential & 3,389 \\
\hline 2-Parameter Exponential & 1,502 \\
\hline Weibull & 0,772 \\
\hline 3-Parameter Weibull & 0,497 \\
\hline Smallest Extreme Value & 2,851 \\
\hline Largest Extreme Value & 0,448 \\
\hline Gamma & 0,474 \\
\hline 3-Parameter Gamma & 0,540 \\
\hline Logistic & 0,976 \\
\hline Loglogistic & 0,235 \\
\hline 3-Parameter Loglogistic & 0,233 \\
\hline Johnson Transformation & 0,183 \\
\hline
\end{tabular}

Fonte: Autores

Os gráficos de probabilidade acima, de maneira visual, permitem o teste de hipóteses pela adequação a determinada distribuição e faz necessário a análise do $\rho$ valor correspondente. Se o valor de $\rho$ for inferior ou igual a $\alpha$ que é o nível de significância e vamos considerar $\alpha=0,05$ então rejeita-se a hipótese nula de que os dados seguem a distribuição. O software Minitab para alguns casos nem sempre converge matematicamente retornando um valor $\rho$ para o teste Anderson-Darling.

Com base nos valores da estatística Anderson-Darling expressos na tabela 6 e pela análise visual dos gráficos da figura 3, verifica-se que a distribuição lognormal apresenta a melhor aproximação para os dados sendo a lognormal a distribuição que melhor modela os dados.

Definida a distribuição da probabilidade como sendo Lognormal aplicamos os valores ao modelo de confiabilidade.

$R(t)=1-\sigma\left(\frac{\ln (t)-\mu}{\sigma}\right)$

$\sigma=0,56858$

$\mu=3,70168$

$R(t)=0,47$

Logo, considerando $t=100$ horas temos $R(t)=0,47$ ou seja para cada 100 horas temos a probabilidade de 47 horas da empacotadora não quebrar.

Calcularemos agora o tempo médio até a falha, sendo:

$M T T F=e^{\mu+0,5 . \sigma^{2}}$

$M T T F=47,62$ horas como o dia de trabalho da linha de salgadinhos corresponde a 19,33 horas, temos um MTTF correspondente a 2,5 dias. 


\section{CONCLUSÃO}

O presente trabalho busca por meio da aplicação técnica dos conceitos de confiabilidade e com a utilização de dados reais apoiar a indústria nacional e demonstrar a importância da integração entre empresas e as universidades com o intuito de desenvolver tecnologia e dar longevidade e maturidade aos processos industriais.

A máquina analisada no processo de empacotamento (empacotadora) apresenta uma confiabilidade na ordem de $47 \%$ o que é muito baixo, assim como um indicador de MTTF na ordem de 2,5 dias. A máquina é operada durante 3 turnos por 6 dias, significando que provavelmente na semana de trabalho haverá duas ocorrências de falha possivelmente mecânica.

\section{REFERÊNCIAS}

${ }^{1}$ KARDEC, Alan; NASCIF, Julio. Manutenção Função Estratégica, $2^{\mathrm{a}}$ edição, $\mathbf{1}^{\mathrm{a}}$ Reimpressão 2004. Editora Quality Mark, Rio de Janeiro, Coleção Manutenção, Abraman.

${ }^{2}$ MOREIRA. D. A. Administração da produção e operações. 2. ed. São Paulo:

Pioneira, 2006.

${ }^{3}$ NOWLAN, F. S.; HEAP, H. F. Reliability-centered Maintenance. Technical Report AD/A066-579, National Technical Information Service, US Department of Comerce, Springfield, Virginia, 1978.

${ }^{4}$ FOGLIATO, Flávio Sanson; José Luís Duarte. Confiabilidade e Manutenção Industrial. Elsevier, Rio de Janeiro, 2009.

${ }^{5}$ SIQUEIRA, Iony Patriota de. Manutenção Centrada na Confiabilidade: Manual de Implementação. 1. ed. Rio de Janeiro: Qualitymark, 2005. 408 p.

${ }^{6}$ Xie M, Goh TN, Kong H. Exponential approximation for maintained Weibull components. Int J Qual Maintenance Engng 2000;6(4):260-9.

${ }^{8}$ GIL, A. C. Como elaborar projetos de pesquisa. 4. ed. São Paulo: Atlas, 2002. 